\title{
Gamma Profile Analysis for Stress, Texture and Grain Size
}

\author{
Bob B. He \\ Bruker AXS Inc, 5465 East Cheryl Parkway, Madison, WI 53711, USA \\ Bob.he@bruker-axs.com
}

Keywords: 2D detector, Residual stress, Texture, Grain size

\begin{abstract}
Two-dimensional X-ray diffraction pattern can be described by the diffraction intensity distribution in both $2 \theta$ and $\gamma$ directions. The $2 \mathrm{D}$ pattern can be reduced to two kinds of profiles: $2 \theta$ profile and $\gamma$-profile. The $2 \theta$-profile can be evaluated for phase identification, crystal structure refinement and many applications with many existing algorithms and software. The $\gamma$-profile contains information on texture, stress, and crystal grain size. This article introduces the concept and fundamental algorithms for stress, texture and crystal size analysis by $\gamma$-profile analysis.
\end{abstract}

\section{Introduction}

The diffracted $\mathrm{x}$-rays from a polycrystalline (powder) sample form a series diffraction cone in 3D space since large numbers of crystals oriented randomly in the space are covered by the incident $\mathrm{x}$ ray beam. Each diffraction cone corresponds to the diffraction from the same family of crystalline planes in all the participating grains. Two-dimensional $\mathrm{x}$-ray diffraction (abbreviated as $\mathrm{XRD}^{2}$ ) pattern from a polycrystalline solid or powder sample can be considered as a cross section of the detecting plane and the diffraction cones [1]. The conic section takes different shapes depending on the detector shape, swing angle to the direct beam and distance from the irradiated volume in the sample. For a detector with flat detecting plane, the conic section may be an ellipse, parabola, or hyperbola. For convenience, all kinds of conic sections will be referred to as diffraction rings or Debye rings without referring to their specific shapes. Figure 1(a) is a diffraction frame collected from a proprietary multilayer battery anode with a $2 \mathrm{D}$ detector. The Bragg angle is denoted by $2 \theta$ and the angle along each diffraction ring can be described by $\gamma$. Figure 1(b) is the same diffraction pattern displayed in 3D plot with intensity in vertical direction. The intensity variation and discontinuity along the diffraction rings can be observed on the diffraction pattern.

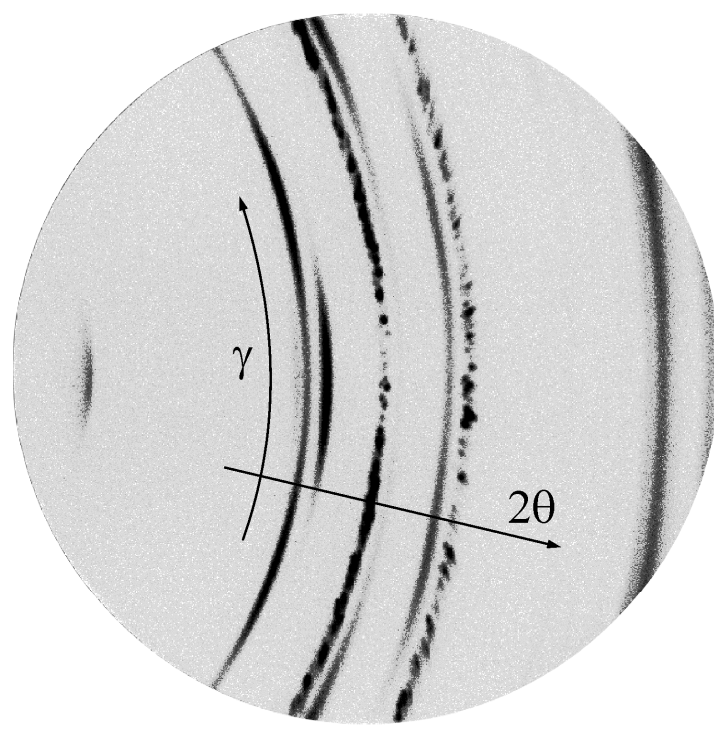

(a)

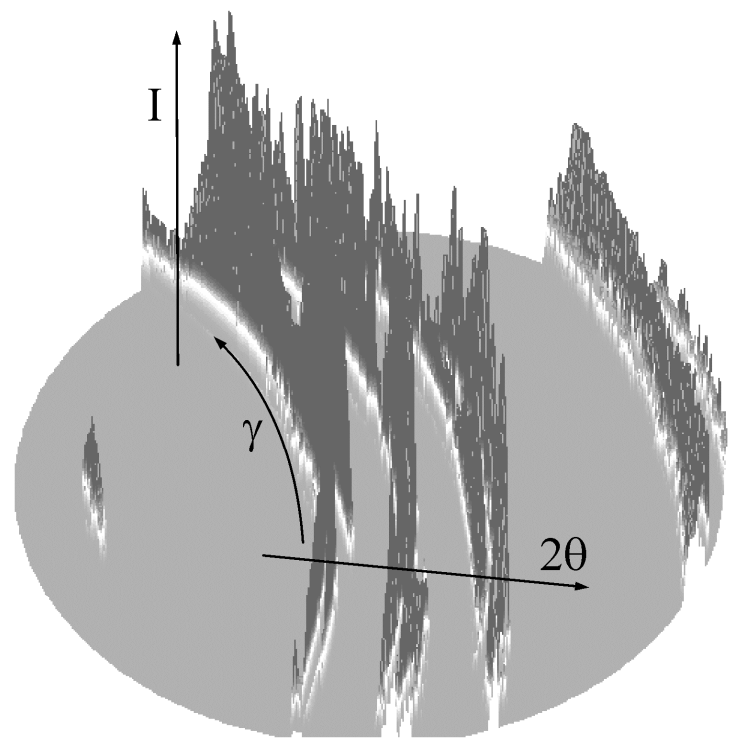

(b)

Figure 1 (a) A 2D diffraction pattern from a battery anode; (b) the 2D pattern displayed in 3D plot with the intensity in vertical direction. 
The $\mathrm{XRD}^{2}$ pattern can be analyzed directly or by data reduction to the intensity distribution along $\gamma$ or $2 \theta$. The $\gamma$-integration can reduce the pattern from a $2 \mathrm{D}$ image into a $1 \mathrm{D}$ diffraction profile analogues to the conventional diffraction pattern which is the diffraction intensity distribution as a function of $2 \theta$ angles. This kind of diffraction pattern can be evaluated by most existing software and algorithms for conventional applications, such as, phase identification, structure refinement and $2 \theta$-profile analysis. However, the materials structure information associated to the intensity distribution along $\gamma$ direction is erased by $\gamma$-integration. In order to evaluate the materials structure associated with the intensity distribution along $\gamma$ angle, either the $2 \mathrm{D}$ diffraction pattern should be directly analyzed or the $\gamma$-profile generated by $2 \theta$-integration should be used.

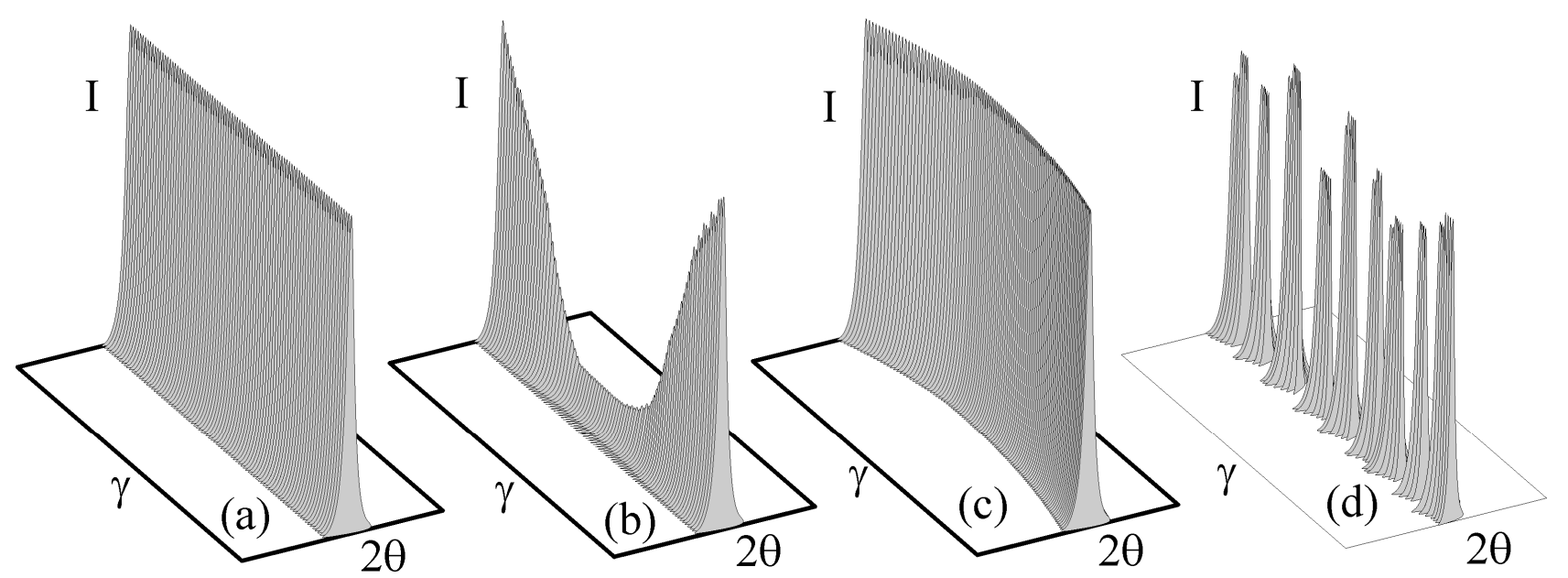

Figure 2. Illustrations of gamma plots from (a) a random fine powder, (b) sample with textured, (c) sample with stress, and (d) a sample with large crystal size.

The diffraction pattern in Figure 1 can be converted to an image with a rectangular $\gamma-2 \theta$ coordinates. This image can be referred to as $\gamma-2 \theta$ plot or simply gamma plot. Figure 2 illustrates four $\gamma-2 \theta$ plots of a single diffraction ring in 3D view. Figure 2(a) shows a straight "wall" with a constant $2 \theta$ and constant intensity along $\gamma$ due to the large amount of random fine powder irradiated by the incident $\mathrm{x}$-ray, so that the number of crystallites satisfying the Bragg condition is statistically the same at all $\gamma$ angles. Figure 2(b) shows the intensity variation along $\gamma$ due to preferred orientation. The texture in the sample will change the diffraction intensity measured at different angle, but not the $2 \theta$ value. Figure 2(c) illustrates the intensity distribution when the sample contains residual stress or under stress loading. The d-spacing variation at different orientation due to stress results in the $2 \theta$ value variation measured at different $\gamma$ angles. Figure 2(d) shows a spotty intensity distribution along $\gamma$ due to only a limited number of large crystals satisfying the Bragg condition. Apparently, the spottiness is related to the crystal size and size distribution. A real material may contain texture, stress and/or large crystal size simultaneously so the $2 \mathrm{D}$ diffraction pattern can be a mix of the above four models.

\section{Diffraction Vector Containing $\gamma$ Variable}

In $\mathrm{XRD}^{2}$ data analysis, the diffraction intensity distribution in both $2 \theta$ and $\gamma$ directions should be considered. In order to include the diffraction intensity variation in $\gamma$ direction, the diffraction vector (also referred to as scattering vector) should include not only the $2 \theta$ variable, but also the $\gamma$ variable. Fig. 3(a) illustrates the diffraction cone and diffraction vector cone in the laboratory coordinates $\mathrm{X}_{\mathrm{L}} \mathrm{Y}_{\mathrm{L}} \mathrm{Z}_{\mathrm{L}}$. The unit vector of the incident beam is $s_{o}$ and the unit vector representing a direction given by $2 \theta$ and $\gamma$ on the diffraction cone is $s$. The diffraction vector $\mathbf{H}$ and its unit vector $\boldsymbol{h}_{\boldsymbol{L}}$ in the laboratory coordinates are given by 


$$
\mathbf{H}=\frac{\mathbf{s}-\mathbf{s}_{0}}{\lambda}=\frac{1}{\lambda}\left[\begin{array}{c}
\cos 2 \theta-1 \\
-\sin 2 \theta \sin \gamma \\
-\sin 2 \theta \cos \gamma
\end{array}\right] \text { and } \mathbf{h}_{L}=\frac{\mathbf{H}}{|\mathbf{H}|}=\left[\begin{array}{l}
\boldsymbol{h}_{\boldsymbol{x}} \\
\boldsymbol{h}_{\boldsymbol{y}} \\
\boldsymbol{h}_{z}
\end{array}\right]=\left[\begin{array}{c}
-\sin \theta \\
-\cos \theta \sin \gamma \\
-\cos \theta \cos \gamma
\end{array}\right]
$$
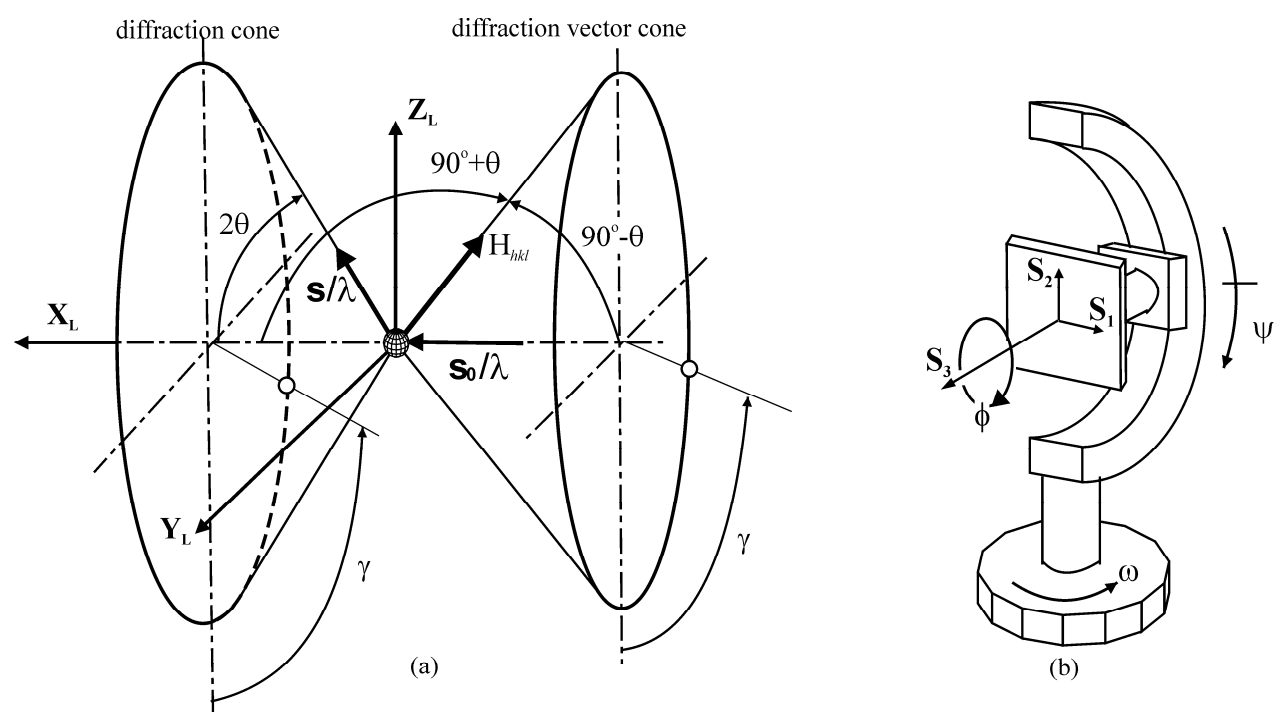

Fig. 3 The diffraction cone and diffraction vector cone (a) and Eulerian geometry (b).

For the Eulerian geometry as shown in Fig. 3(b) with three sample rotation angles $(\omega, \psi, \phi)$, the three components of the unit diffraction vector in the sample coordinates $\left(S_{1}, S_{2}, S_{3}\right)$ are given by:

$$
\begin{gathered}
\boldsymbol{h}_{1}=\sin \theta(\sin \phi \sin \psi \sin \omega+\cos \phi \cos \omega)+\cos \theta \cos \gamma \sin \phi \cos \psi-\cos \theta \sin \gamma(\sin \phi \sin \psi \cos \omega-\cos \phi \sin \omega) \\
\boldsymbol{h}_{2}=-\sin \theta(\cos \phi \sin \psi \sin \omega-\sin \phi \cos \omega)-\cos \theta \cos \gamma \cos \phi \cos \psi+\cos \theta \sin \gamma(\cos \phi \sin \psi \cos \omega+\sin \phi \sin \omega) \\
\boldsymbol{h}_{3}=\sin \theta \cos \psi \sin \omega-\cos \theta \sin \gamma \cos \psi \cos \omega-\cos \theta \cos \gamma \sin \psi
\end{gathered}
$$

It will be shown in the following section that, many important equations can be derived from the above expression of the unit diffraction vector in the sample coordinates.
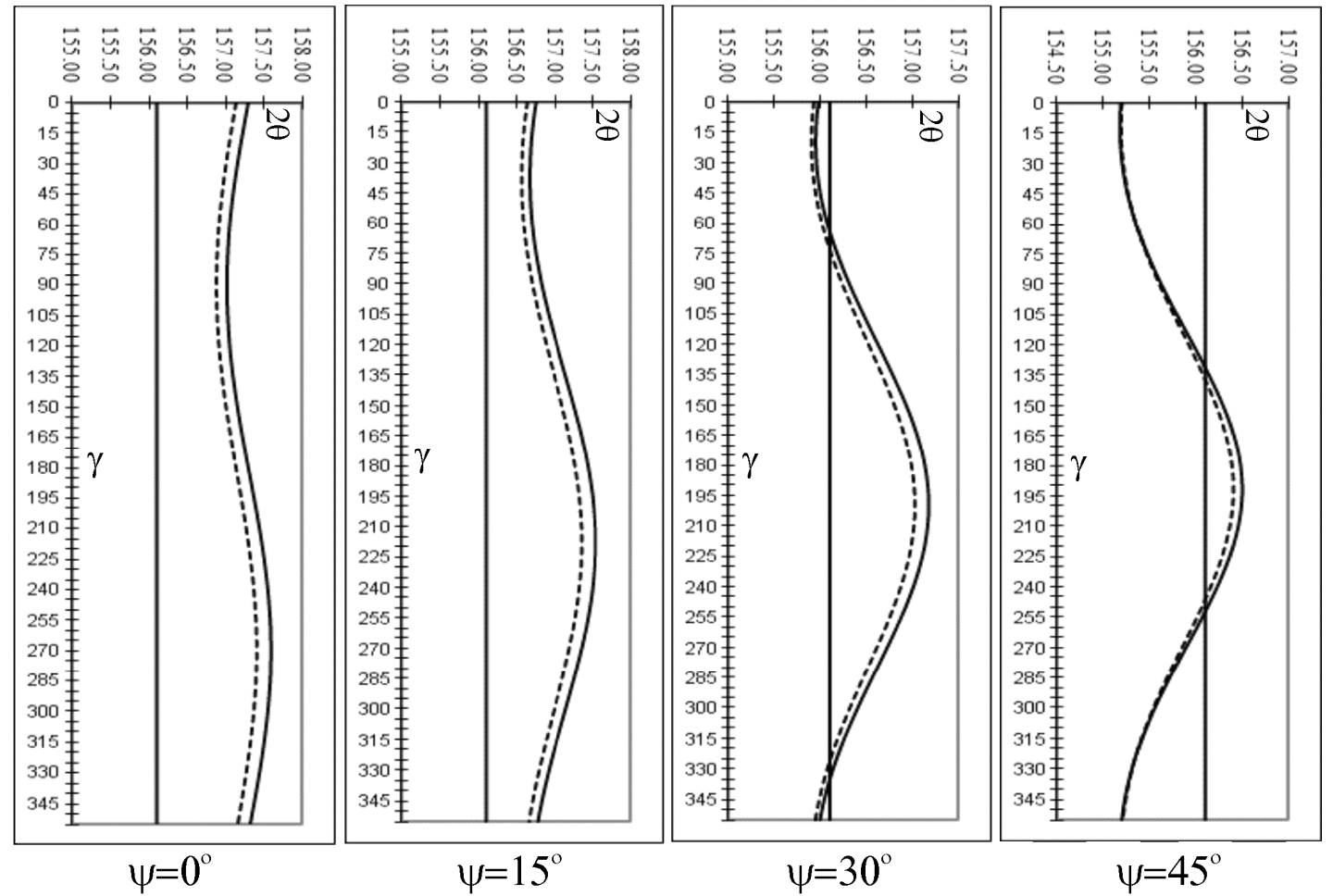

Figure $4.2 \theta$ vs. $\gamma$ plots for steel with $\sigma_{11}=\sigma_{22}=1000 \mathrm{MPa}$ at various $\psi$ angles. 


\section{Stress}

The $\sin ^{2} \psi$ method is mostly used for stress measurement with the conventional X-ray diffractometer which uses either a point detector or line detector [2]. The fundamental equation for stress measurement with $\mathrm{XRD}^{2}$ can be given as [3]:

$$
\boldsymbol{S}_{1}\left(\sigma_{11}+\sigma_{22}+\sigma_{33}\right)+\frac{1}{2} \boldsymbol{S}_{2}\left(\sigma_{11} \boldsymbol{h}_{1}^{2}+\sigma_{22} \boldsymbol{h}_{2}^{2}+\sigma_{33} \boldsymbol{h}_{3}^{2}+2 \sigma_{12} \boldsymbol{h}_{1} \boldsymbol{h}_{2}+2 \sigma_{13} \boldsymbol{h}_{1} \boldsymbol{h}_{3}+2 \sigma_{23} \boldsymbol{h}_{2} \boldsymbol{h}_{3}\right)=\ln \left(\frac{\sin \theta_{0}}{\sin \theta}\right)
$$

where $S_{1}$ and $\frac{1}{2} S_{2}$ are the macroscopic elastic constants, and $h_{1}, h_{2}$ and $h_{3}$ are the three components of the unit diffraction vector. By taking various $\gamma$ value, it defines the $2 \theta$ value variation measured at different $\gamma$ angles for a particular stress tensor and sample orientation. Figure 4 shows the simulated $2 \theta$ shift as a function $\gamma$ for carbon steel with equibiaxial stress $\sigma_{11}=\sigma_{22}=1000 \mathrm{MPa}$. The straight solid line represents the stress free $2 \theta=156.1^{\circ}$. The goniometer angles are set at $\omega=78^{\circ}$ and $\phi=0^{\circ}$. The four plots from left to the right are for $\psi=0^{\circ}, 15^{\circ}, 30^{\circ}$ and $45^{\circ}$ respectively. The solid curves represents $2 \theta$ shift based on isotropic elasticity and the dotted curves are based on anisotropic factor $\mathrm{A}_{\mathrm{RX}}=1.49[4]$.

\section{Texture}

For a sample with texture, the diffraction intensity becomes a function of the sample orientation and $\gamma$ value. Fig. 5 shows the pole density distribution in a pole figure corresponding to the intensity variation along $\gamma$ from a single $2 \mathrm{D}$ pattern. The pole density at $(\alpha, \beta)$ is proportional to the integrated intensity at the same angles [4]:

$$
\boldsymbol{P}_{h k l}(\alpha, \beta)=\boldsymbol{K}_{h k l}(\alpha, \beta) \cdot \boldsymbol{I}_{h k l}(\alpha, \beta)
$$

where $\boldsymbol{I}_{h k l}(\alpha, \beta)$ is the $2 \theta$-integrated intensity of the $(h k l)$ peak corresponding to the pole direction $(\alpha, \beta), \quad \boldsymbol{K}_{h k l}(\alpha, \beta)$ is the scaling factor covering the absorption, polarization, background corrections and various instrument factors if these factors are included in the integrated intensities, and $\boldsymbol{P}_{h k l}(\alpha, \beta)$ is the pole density distribution function. The pole figure angles $(\alpha, \beta)$ can be calculated from the unit diffraction vector components by the following pole mapping equations:

$$
\alpha=\sin ^{-1}\left|\boldsymbol{h}_{3}\right|=\cos ^{-1} \sqrt{\boldsymbol{h}_{1}^{2}+\boldsymbol{h}_{2}^{2}} \text { and } \beta= \pm \cos ^{-1} \frac{\boldsymbol{h}_{1}}{\sqrt{\boldsymbol{h}_{1}^{2}+\boldsymbol{h}_{2}^{2}}} \quad\left\{\begin{array}{lll}
\beta \geq 0^{\circ} & \text { if } & \boldsymbol{h}_{2} \geq 0 \\
\beta<0^{\circ} & \text { if } & \boldsymbol{h}_{2}<0
\end{array}\right.
$$

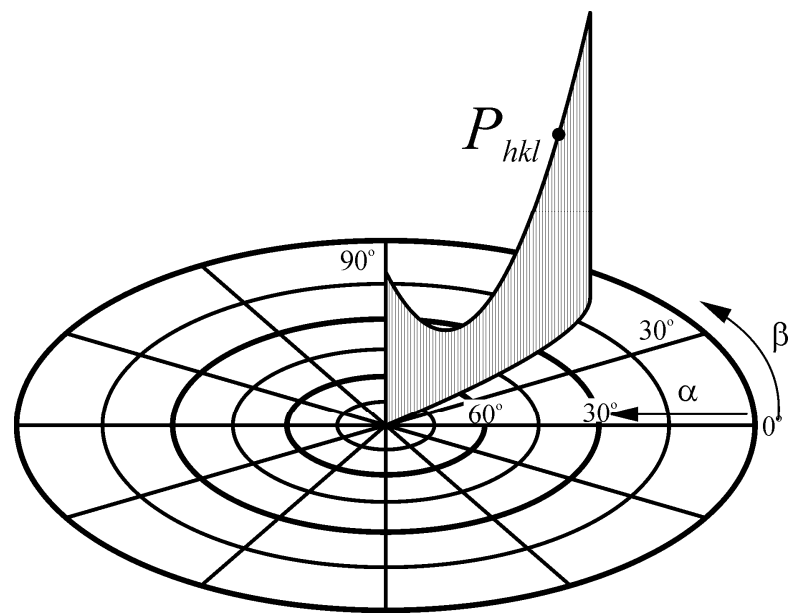

Figure 5. Pole density mapping from $\gamma$ profile to pole-figure 


\section{Crystal size}

The size of the crystallites in a polycrystalline material has significant effects on many of its properties, such as thermal, mechanical, electrical, magnetic and chemical properties. X-ray diffraction has been used for crystallites size measurement for over ninety years based on diffraction line broadening and line profile analysis of the convention $2 \theta$ profile. Figure 6 (a) shows diffraction profile collected from gold nano-particles and regular gold metal. The $2 \theta$ profile from the gold nano-particle is significantly broader than the profile from regular gold metal. Negliecting other sources of broadening, such as microstrains, the crystallite size can be measured from the broadening by Scherrer equation, The $2 \theta$ profile analysis is suitable only for crystallite sizes below $100 \mathrm{~nm}$. The $\gamma$ profile analysis can significantly extend measurable range up to millimeters. The detailed theory can be found in the reference [1]. It can be simply explained by a candy box. The number of candies to fill the box depends on the size of the candy and candy box. The effective diffraction gauge volume can be considered as a "candy box". The number of spots along the diffraction ring in a given $\gamma$ range is associated to the crystallite size (also referred as gain size or particle size in some articles). Figure 6(b) shows a frame collected from a LaB6 sample. The spotty diffraction rings are observed. Based on the effective diffraction volume and the crystallographic nature of the sample, in reflection mode diffraction, the crystal size is given by:

$$
\boldsymbol{d}=\boldsymbol{k}\left\{\frac{\boldsymbol{p}_{h \boldsymbol{k}} \boldsymbol{b}^{2} \arcsin [\cos \theta \sin (\Delta \gamma / 2)]}{2 \mu \boldsymbol{N}_{s}}\right\}^{1 / 3}
$$

where $\boldsymbol{d}$ is the average diameter of the crystallite, $\boldsymbol{p}_{\boldsymbol{h} \boldsymbol{k} \boldsymbol{l}}$ is the multiplicity of the diffracting planes, $\boldsymbol{b}$ is the size of the incident beam, $\mu$ is the linear absorption coefficient and $N_{s}$ is the number of diffraction spots within the measured range $\Delta \gamma$ of the diffraction ring. The scaling factor $\boldsymbol{k}=(3 \beta / 4 \pi)^{1 / 3}$, in which $\beta$ is the divergence of the incident X-ray beam. But we may treat $\boldsymbol{k}$ as a calibration factor covering all the numeric constants, incident beam divergence and other instrument conditions, which can be determined from the 2D diffraction pattern of a known standard. Since only a limited number of spots along the diffraction ring can be resolved, it can be seen that smaller $\mathrm{x}$-ray beam size and low multiplicity peak should be used if a smaller crystallite size is to be determined. For measurement in transmission mode with a sample thickness of $\boldsymbol{t}$, we have:

$$
\boldsymbol{d}=\boldsymbol{k}\left\{\frac{\boldsymbol{p}_{h k l i} \boldsymbol{b}^{2} \boldsymbol{t} \arcsin [\cos \theta \sin (\Delta \gamma / 2)]}{\boldsymbol{N}_{s}}\right\}^{1 / 3}
$$

It can be observed from the above equations that the effective sampling volume is proportional to the beam cross section area and is lower for materials with higher linear absorption coefficients. The sample absorption has a different effect on the sampling volume in reflection and transmission modes. In reflection mode, the linear absorption coefficient determines how fast the incident beam is attenuated within the sample, but there is no clear cutoff for the penetration depth. Therefore, the effective sampling volume has to be used. In transmission mode with the incident beam perpendicular to the sample surface, the linear absorption coefficient affects the relative scattering intensity (counting statistics), but not the actual sampling volume. In other words, all the sample volume within the beam path contributes to the diffraction. Therefore, it is reasonable to ignore the absorption effect for crystallite size analysis as long as the sample is thin enough for transmission mode diffraction.

Figure 6(c) shows the measurement range of the crystal size analysis by the conventional $2 \theta$ profile methods and the $\gamma$ profile method. The $2 \theta$ profile analysis is suitable for crystal size below $100 \mathrm{~nm}$, while $\gamma$ profile analysis is suitable for crystal size from sub-micrometer to a few millimeters. The size range of $\gamma$ profile analysis can be further extended by instrumentation and data collection strategy. 


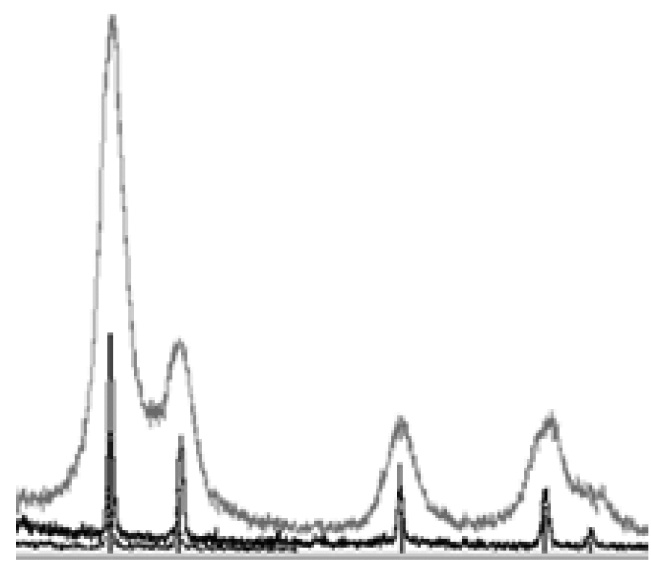

$2 \theta$

(a)

(b)

(111)

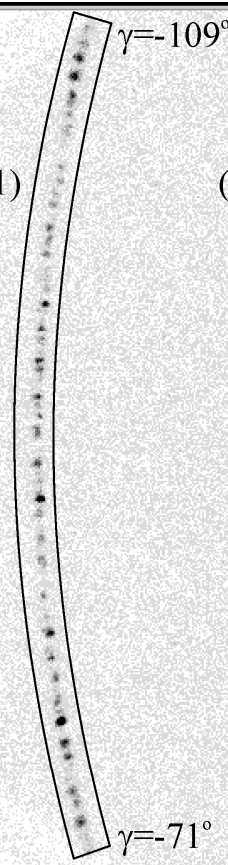

(110)

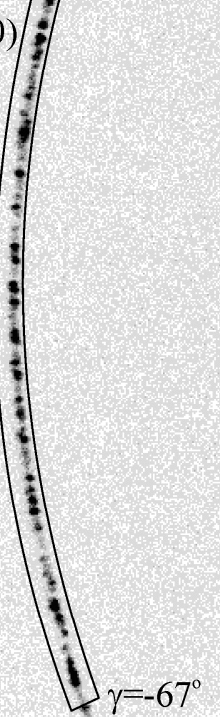

(100)

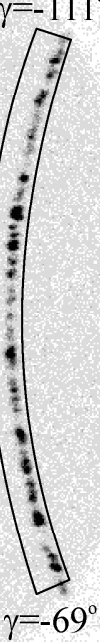

$\gamma$ profile analysis

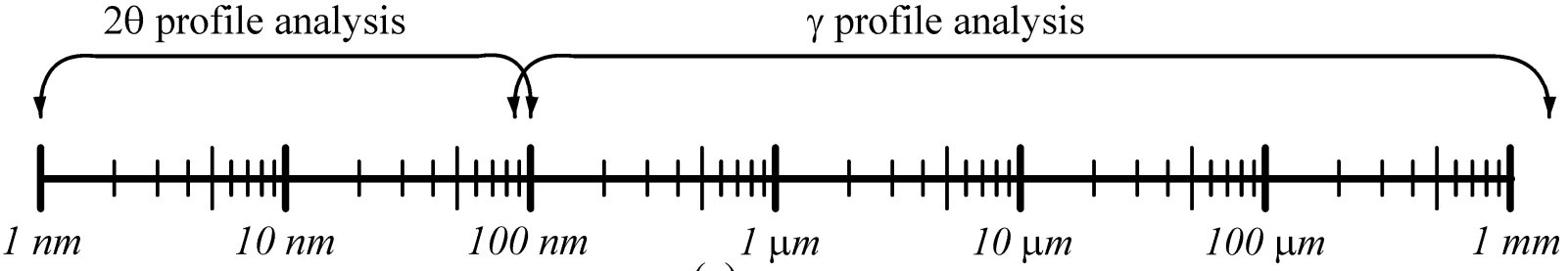

(c)

Figure 6. Crystal size measurement range of $2 \theta$ profile and $\gamma$ profile analysis.

\section{Conclusions}

Two-dimensional X-ray diffraction pattern can be described by the diffraction intensity distribution in both $2 \theta$ and $\gamma$ directions. In order to evaluate the materials structure associated with the intensity distribution along gamma angle, either the $\mathrm{XRD}^{2}$ pattern should be directly analyzed or the $\gamma$-profile can be generated by $2 \theta$-integration. A gamma profile may contain information on texture, stress, and crystal size. The diffraction intensity distribution in $\gamma$ direction can be used for materials characterization. Preferred orientation causes intensity variation in $\gamma$ direction. Residual stress results in $2 \theta$ shift as a function of $\gamma$. Spotty intensity distribution in $\gamma$ direction is associated to crystal size.

\section{References}

[1] B. B. He, Two-dimensional X-ray Diffraction, John Wiley \& Sons, Hoboken, New Jersey 2009.

[2] I. C. Noyan and J. B. Cohen, (1987). Residual Stress, Spinger-Verlag, New York 1987.

[3] B. B. He, U. Preckwinkel and K. L. Smith, Advantages of Using 2D Detectors for Residual Stress Measurement, Advances in X-ray Analysis, Vol. 42, pp. 429-438, 1998.

[4] B. B. He and K. L. Smith, Computer simulation of diffraction stress measurement with 2D detectors, Proceedings of 1998 SEM Spring Conference, Houston, USA.

[5] H. J. Bunge and H. Klein, Determination of quantitative, high-resolution pole figures with the area detector, Z. Metallkd. Vol. 87, No. 6, pp. 465-475, 1996. 